34 Kliks BR, Burgess $M$, Abildskov J. Influence of sympathetic tone on ventricular fibrillation threshold during experimental coronary occlusion. Am f Cardiol 1975;36: 45-9.

35 Brown G, Lee A, Bolson E, Dodge H. Reflex constriction of significant coronary stenosis as a mechanism contributing to ischaemic left ventricular dysfunction during isometric exercise. Circulation 1984; 70:18-24.

36 Vatner S, Hintze T, Macho P. Regulation of large coronary arteries by beta adrenergic mechanisms in the conscious dog. Circ Res 1982;51:56-66.

37 Chierchia S, Lazzari M, Freedman B, Brunelli C, Maseri A. Impairment of myocardial perfusion and function during painless myocardial ischaemia. $\mathcal{F} \mathrm{Am}$ Coll Cardio 1983;1:924-30.

38 Shakespeare CF, Crowther A, Cooper IC, Katritsis D, Coltart DJ, Webb-Peploe MM. Autonomic reflexes initiated by balloon angioplasty [abstract]. Circulation $1991 ; 84($ II $): 268$
Department of

Cardiology and

Cardiovascular

Surgery, Royal

Hospital, Muscat,

Sultanate of Oman

R Shahrabani

R Chakraborty

J Valliathu

Correspondence to

Dr Rashid M Shahrabani,

Department of Cardiology,

Royal Hospital,

P. O. Box 1331 Seeb,

Sultanate of Oman.

Accepted for publication

20 September 1993

\section{Cross sectiona}

echocardiograms in the

long axis parasternal view

showing $(A)$ a dilated,

unruptured aneurysm of

the right sinus of Valsalva

dissecting into the upper

part of interventricular

septum and $(B)$ complete

obliteration of the

aneurysm in the

interventricular septum

after operation.

$A O$, aorta; $L V$, left

ventricle; RCS, right

coronary sinus; $R V$, right

ventricle.

\title{
Acute pulmonary oedema: an unusual clinical presentation of unruptured sinus of Valsalva
}

\author{
Rashid Shahrabani, Rabindranath Chakraborty, John Valliathu
}

A 24-year-old man was admitted with a 5 -day history of giddiness, a cough with whitish expectoration, breathlessness, and orthopnoea. The patient showed no marfanoid features. He was afebrile but dyspnoeic. His pulse (40 beats/min) was regular and he showed raised jugular venous pulsation with visible cannon waves. Blood pressure was $130 / 60 \mathrm{~mm} \mathrm{Hg}$. At auscultation an early diastolic murmur (grade 2/6) was heard at the lower sternal edge. An electrocardiogram showed complete heart block, and a chest $x$ ray taken with portable equipment showed cardiomegaly and frank pulmonary oedema. All haematological and biochemical variables were normal. Three blood cultures were negative. Cross sectional
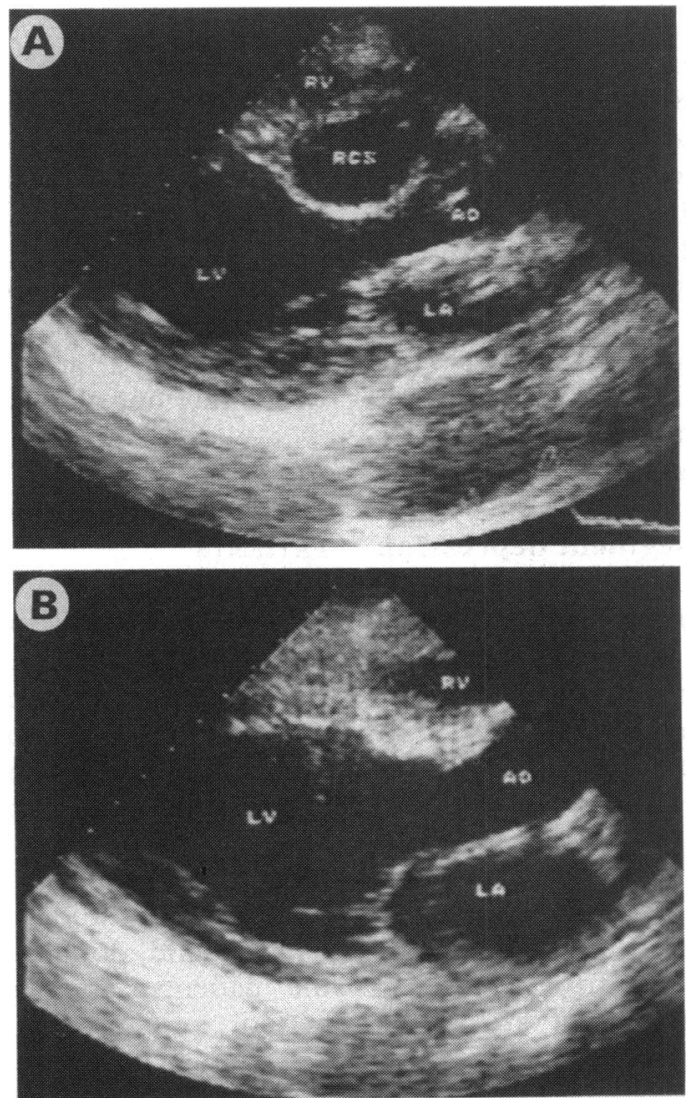

echocardiography confirmed the diagnosis of a dilated, unruptured aneurysm of the right coronary sinus dissecting into the interventricular septum and producing a cystic mass (fig A). Cardiac catheterisation confirmed the presence of an unruptured aneurysm of the right sinus of Valsalva dissecting into the upper part of the interventricular septum with grade $2 / 6$ aortic regurgitation. There was no gradient across the outflow tract. The results of selective coronary angiography were normal.

The patient was treated for heart failure with intravenous frusemide and supplementary potassium and a transvenous temporary pacing wire was inserted. He underwent cardiopulmonary surgery. The ostium of the aneurysm was closed with Dacron patch and a permanent pacemaker was implanted. Postoperatively cross sectional echocardiography showed signs of mild aortic regurgitation. The cystic cavity in the septum had been completely obliterated (fig B).

Unruptured aneurysm of sinus of Valsalva is a rare anomaly that commonly affects the right coronary sinus. ${ }^{1}$ The aneurysm may present clinically as aortic regurgitation ${ }^{2}$ or as a mediastinal mass, ${ }^{3}$ cerebrovascular accident, ${ }^{4}$ or complete heart block. ${ }^{5}$ Some patients have presented with dyspnoea on exertion (NYHA functional class II)..$^{56}$ Our patient was orthopnoeic (NYHA functional class IV) with clinical and radiological features of frank pulmonary oedema. His condition improved considerably after successful surgical repair.

1 Fisbein MC, Obma R, Roberts WC. Unruptured sinus of Valsalva aneurysm. Am f Cardiol 1975;24:918-22.

2 London SB, London RE: Production of aortic regurgitation by unperforated aneurysm of sinus of Valsalva. Circulation 1961;24:1403-6.

3 Reid PG, Goudevenos JA, Hilton CJ. Thrombosed saccular aneurysm of a sinus of Valsalva: Unusual cause of a mediastinal mass. Br Heart $\mathcal{F} 1990 ; 63: 183-5$.

4 Shahrabani R, Jairaj PS. Unruptured aneurysm of the sinus of Valsalva: a potential source of cerebrovascular embolism. Br Heart $\mathcal{f}$ 1993;69:266-7.

5 Ahmed RAS, Struman S, Watson RDS. Unruptured aneurysm of the sinus of Valsalva presenting with isolated heart block: echocardiographic diagnosis and successful surgical repair. Br Heart $\mathcal{f} 1989 ; 61: 375-7$.

6 Metras D, Coulibaly AO, Ouattara K. Calcified unruptured aneurysm of sinus of Valsalva with complete heart block and aortic regurgitation. Br Heart $\mathcal{f} 1982 ; 48$ : 507-9. 